\title{
Accelerated and Safe Proliferation of Human Adipose-derived Stem Cells in Medium Supplemented with Human Serum
}

\author{
Fonny Josh ${ }^{1}$, Kyoko Kobe ${ }^{2}$, Morikuni Tobita ${ }^{1}$, Rica Tanaka ${ }^{1}$, \\ Koji Suzuki, Kasumi Ono ${ }^{3}$, Hiko Hyakusoku² and Hiroshi Mizuno ${ }^{1,2}$ \\ ${ }^{1}$ Department of Plastic and Reconstructive Surgery, Juntendo University School of Medicine \\ ${ }^{2}$ Department of Plastic and Reconstructive Surgery, Nippon Medical School \\ ${ }^{3}$ R\&D Central Research Laboratory, JMS Co. Ltd., Hiroshima
}

\begin{abstract}
Adipose-derived stem cells (ASCs) are a promising cell source and are being investigated for a variety of therapeutic applications. However, standard expansion protocols use fetal bovine serum (FBS) as a growth factor supplement, which is a potential source of undesirable xenogeneic pathogens. For clinical safety, autologous human serum (HS) would be more appropriate. This study compared FBS-supplemented and HS-supplemented media for their enhancement of the proliferation and differentiation potential of human ASCs (hASCs). HS was obtained from the blood of 8 healthy volunteers using collection devices specially designed to derive growth factors from platelets. Growth factors in HS or FBS were measured with enzyme-linked immunosorbent assays. The hASCs were isolated with an established protocol from discarded human fat tissues obtained during a medical procedure and cultured in a medium supplemented with either 10\% HS or 10\% FBS. The hASCs were collected at several time points for the proliferation assays. The capacity for differentiation into the osteogenic, chondrogenic and adipogenic lineages was assessed qualitatively with the histochemical stains von Kossa, Alcian blue, and Oil red O, respectively, and quantitatively with the qualitative reverse transcriptase polymerase chain reaction. Differences in cell surface marker expression between the HS-supplemented and FBS-supplemented cultures were examined with flow cytometric analysis. Proliferation assays showed that the growth of hASCs was more rapid in HS-supplemented medium than in FBS-supplemented medium. All cells grown in each medium expressed similar patterns of cell surface markers. The ASCs cultured in the HS-supplemented medium proliferated more rapidly than those cultured in the FBS-supplemented medium and retained their differentiation capacity and immunophenotype. These results support the establishment of a safe and rapid expansion protocol with autologous serum for cell-based therapies, such as tissue engineering and regenerative medicine, using hASCs.
\end{abstract}

(J Nippon Med Sch 2012; 79: 444-452)

Key words: adipose-derived stem cells, human serum, mesenchymal stromal cells, fetal bovine serum, platelet-derived factors

Correspondence to Hiroshi Mizuno, MD, PhD, Department of Plastic and Reconstructive Surgery, Juntendo University School of Medicine, 2-1-1 Hongo, Bunkyo-ku, Tokyo 113-8421, Japan

E-mail: hmizuno@juntendo.ac.jp

Journal Website (http://www.nms.ac.jp/jnms/) 


\section{Introduction}

Cell therapy has received much attention owing to the ability of stem/progenitor cells to regenerate damaged tissues and organs. To date, many cell types have been presented as promising candidates for regenerative cell therapy, including embryonic stem cells, induced pluripotent stem cells ${ }^{1}$, and stem/ progenitor cells from adult tissues ${ }^{2}$. Despite the excellent potential of embryonic stem cells, there are many ethical issues concerning their use ${ }^{3}$. The technology to develop induced pluripotent stem cells may overcome these ethical issues without sacrificing multipotency. However, there are still some limitations to their practical use, such as cell regulation and genetic manipulation ${ }^{1,4,5}$.

Mesenchymal stem cells are multipotent, nonhematopoietic stem cells that are typically obtained from bone marrow ${ }^{6}$ but can also be isolated from several other tissues, such as umbilical cord blood $^{7}$ and adipose tissue ${ }^{8-11}$.

Because subcutaneous adipose tissue is abundant and readily accessible ${ }^{12}$, it is a promising source of stem cells. Despite these advantages, adiposederived stem cells (ASCs) might need to be grown in vitro, depending on the circumstances. For example, in the case of cell-based therapy, ASCs must be cultivated and grown safely and rapidly, while their multipotency is maintained.

Common protocols for clinical trials and basic research employing human ASCs (hASCs) use a growth medium supplemented with fetal bovine serum $(\mathrm{FBS})^{13}$. However, FBS might be an undesirable source of xenogeneic antigens and might transmit zoonoses, such as animal viruses or prions. Additionally, FBS has been implicated in anaphylactic or Arthus-like immune reactions in patients who received cells generated in an FBSsupplemented medium ${ }^{14,15}$ and can even lead to arrhythmias after cellular cardioplasty ${ }^{16}$. For clinical use, hASCs should ideally be prepared with autologous serum. Few reports have demonstrated how human serum (HS) affects hASC proliferation and differentiation ${ }^{17-20}$.

Platelets provide many kinds of growth factors

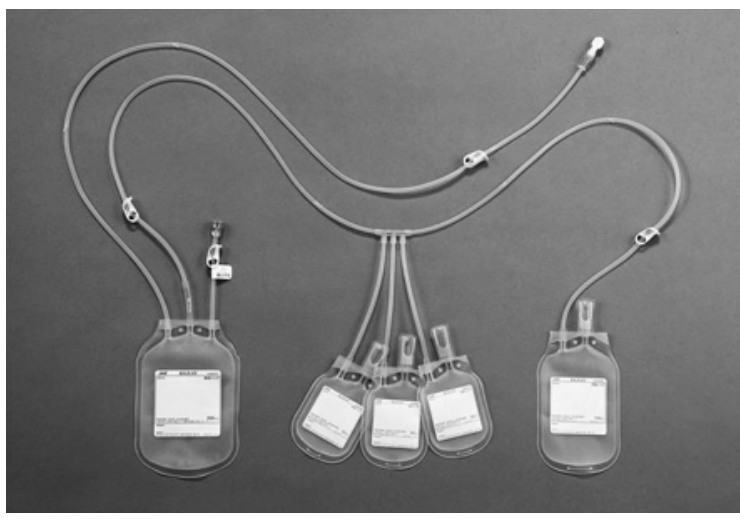

Fig. 1 Appearance of Cellaid $^{\circledR}$, a closed-system serum-collecting bag. The bags contain glass beads for platelet activation. The beads promote blood coagulation and release of growth factors.

that stimulate cell proliferation ${ }^{18,21,22}$. A recently developed serum collection bag was specially designed to activate platelets and derive growth factors from them, and the efficacy of such preparations in stimulating cell proliferation has been confirmed with several cell types ${ }^{23-25}$. The aim of the present study was to investigate the efficacy of HS in preserving the proliferation and differentiation capacity of ASCs in cell culture.

\section{Materials and Methods}

\section{Preparation of HS}

Cellaid $^{\circledR}$ (JMS Co., Ltd., Hiroshima, Japan), which is a completely closed serum-collecting bag system for separating serum for cell cultivation (Fig. 1), was used in these studies. The system is specially designed for collecting serum from whole blood, while activating platelets and removing fibrin. Twohundred-milliliter samples of venous whole blood from 8 healthy donors ( 7 men and 1 woman; average age, 35.5 years; age range, 29 to 44 years) were collected in a large bag containing glass beads. The blood was then incubated at room temperature for 30 minutes with constant gentle agitation that allowed platelets to attach to the glass beads and release endogenous growth factors. Approximately $90 \mathrm{~mL}$ of HS from each donor was then separated with centrifugation at 2,500 rpm for 10 minutes. The serum was isolated, heat-inactivated at $56^{\circ} \mathrm{C}$ for 30 
minutes, and stored at $-80^{\circ} \mathrm{C}$ until use $e^{23,24}$.

\section{hASC Culture}

Human subcutaneous fat was obtained from excess tissues excised during elective plastic surgery from 4 patients, with their written consent and that of their family. Tissue samples were used with the approval and according to the guidelines of the Institutional Review Board of Nippon Medical School based on the Declaration of Helsinki (Approval Number: 19-11-36). The hASCs were harvested and processed according to our established protocols ${ }^{26-28}$. Briefly, the fat tissue was excised and extensively washed with phosphatebuffered saline (Gibco-BRL, Grand Island, NY, USA). The tissue was then finely minced, digested in $0.15 \%$ collagenase (Wako Pure Chemical Industries, Ltd., Osaka, Japan), and vigorously shaken for 30 minutes at $37^{\circ} \mathrm{C}$ in a $50-\mathrm{mL}$ centrifuge tube. Next, an equal volume of either FBS-supplemented control medium (FBS-CM) containing Dulbecco's Modified Eagle Medium (DMEM, Gibco-BRL), 1\% antibioticantimycotic (Gibco-BRL), and 10\% FBS (Gibco-BRL) or HS-supplemented control medium (HS-CM) (DMEM, 1\% antibiotic-antimycotic, and 10\% HS) was added to neutralize the collagenase. The cell suspensions were then centrifuged at 1,300 rpm (260 $g$ ) for 5 minutes, after which the cell pellets were resuspended in each control medium. The ASCs were plated in 6 -well tissue culture plates $\left(2 \times 10^{5}\right.$ cells/well) and maintained in each medium at $37^{\circ} \mathrm{C}$ in $5 \% \mathrm{CO}_{2}$. The medium was replaced every 3 days. Once the hASCs had reached $80 \%$ to $90 \%$ confluence, they were detached from the culture dishes with $0.25 \%$ trypsin/EDTA (Gibco-BRL), neutralized with a control medium, and then passaged at a dilution of $1: 3$. To exclude differences due to lot-to-lot variability of FBS, 3 different lots of FBS were used.

\section{Determination of Levels of Platelet-derived} Growth Factor and Transforming Growth Factor$\beta$ in HS and FBS

The HS obtained with Cellaid ${ }^{\circledR}$ and the FBS were assayed to quantify the amounts of representative growth factors. Enzyme-linked immunosorbent assays (ELISAs; all from R\&D Systems, Inc., Minneapolis, MN, USA) for human transforming growth factor (TGF)- $\beta 1$ (Quantikine DB100), plateletderived growth factor (PDGF)-AB (Quantikine DHD00B), and PDGF-BB (Quantikine DBB00) were performed for all samples of serum, HS $(n=8)$, and FBS ( $n=3$ different lots).

\section{Cell Proliferation Assay}

Second-passage hASCs were seeded at a density of $1.0 \times 10^{3}$ cells/well in 96-well culture plates. The cells were cultured with either FBS-CM or HS-CM and then collected at 2, 4, 6, 8, and 10 days after incubation. The numbers of cultured cells at each time point were measured with the CellTiter 96 AQueous One Solution Cell Proliferation Assay (Promega Corp., Madison, WI, USA) according to the manufacturer's instructions.

\section{Flow Cytometric Analysis}

At passage 6, the hASCs were detached with $0.25 \%$ trypsin/EDTA treatment. Aliquots of cell suspension containing $1 \times 10^{5} \mathrm{hASC}$ were incubated with antibodies dissolved in phosphate-buffered saline containing $3 \% \mathrm{FBS}$ and $0.05 \% \mathrm{NaN}_{3}$ for 30 minutes on ice. Phycoerythrin-conjugated antibodies against the following cell surface markers were used: CD31, CD34, CD45, CD73, CD90 (BD Bioscience, Franklin Lakes, NJ, USA), and CD105 (eBioscience, Inc., San Diego, CA, USA). Flow cytometry was performed with a FACS-Calibur instrument (BD Japan, Tokyo, Japan) equipped with CellQuest software.

\section{Differentiation Capacity of hASCs in HS-CM}

Assays to induce differentiation into adipogenic, osteogenic, and chondrogenic lineages were carried out at the second cell passage.

For the adipogenic lineage, hASCs were seeded into 24-well culture plates at a density of $1 \times 10^{5}$ cells/well and cultured in either HS-CM or FBS-CM overnight. The cells were then incubated in DMEM supplemented with $1 \mu \mathrm{M}$ dexamethasone (SigmaAldrich Co., St. Louis, MO, USA), $10 \mu \mathrm{M}$ of insulin (Wako, Osaka), $200 \mu \mathrm{M}$ of indomethacin (SigmaAldrich), and $0.5 \mathrm{mM}$ isobutyl-methylxanthine 
(Sigma-Aldrich), and either $10 \%$ HS or $10 \%$ FBS. The medium in each well was replaced twice a week. Two weeks later, the hASCs were fixed in $10 \%$ neutral buffered formalin for 25 minutes and stained with Oil red $\mathrm{O}$.

For differentiation into the osteogenic lineage, hASCs were harvested and seeded at a density of 1 $\times 10^{4}$ cells/well in 24-well plastic culture plates with either HS-CM or FBS-CM. The following day, the cells were incubated in DMEM supplemented with $0.1 \mu \mathrm{M}$ dexamethasone (Sigma-Aldrich), $50 \mu \mathrm{g} / \mathrm{mL}$ ascorbic acid-2-phosphate (Sigma-Aldrich), and either $10 \%$ HS or $10 \%$ FBS. The medium was replaced twice per week. Three weeks later, mineral deposition was identified with von Kossa staining.

Chondrogenic differentiation was induced with the micromass culture technique ${ }^{29}$. Briefly, $10 \mu \mathrm{L}$ of a concentrated hASC suspension $\left(3 \times 10^{6}\right.$ cells $\left./ \mathrm{mL}\right)$ was placed in the center of each well of a 24 -well culture plate and allowed to attach at $37^{\circ} \mathrm{C}$ in $5 \%$ $\mathrm{CO}_{2}$ for 5 hours. Then, DMEM supplemented with $10 \mathrm{ng} / \mathrm{mL} \quad$ TGF- $\beta 1 \quad$ (Sigma-Aldrich), $\quad 6.25 \mu \mathrm{g} / \mathrm{mL}$ insulin (Sigma-Aldrich), $\quad 6.25 \mu \mathrm{g} / \mathrm{mL}$ transferrin (Sigma-Aldrich), and either $10 \%$ HS or $10 \%$ FBS was gently overlaid without detaching the cell clusters, and the medium was changed every 3 days. The cultures were maintained for 3 weeks. Chondrogenesis was confirmed by the presence of proteoglycans identified with Alcian blue staining.

\section{Quantitative Real Time-Polymerase Chain Reaction}

Total RNA was extracted from cultures undergoing adipogenic, osteogenic, or chondrogenic differentiation using the Trizol reagent (Gibco-BRL) according to the manufacturer's protocol.

To quantify the expression level of a target gene in the trilineage differentiation at each time point, the quantitative real time-polymerase chain reaction (qRT-PCR) was performed with complementary DNA as a template. The genes of interest were thise for peroxisomal proliferator-activated receptor (PPAR) $\gamma$ for adipogenic differentiation, runt-related transcription factor (RUNX) 2 and sparc/osteonectin, cwcv and kazal-like domains proteoglycan (SPOCK) 1 for osteogenic differentiation, Sry-related HMG box
(SOX) 9 for chondrogenic differentiation, and human 18S ribosomal RNA as a housekeeping gene (Table 1). Total cellular RNA was isolated and reversetranscribed with the TaqMan Gold RT-PCR kit for real-time PCR (Applied Biosystems, Carlsbad, CA USA). Quantitative RT-PCR was performed with this kit according to the manufacturer's instructions and an ABI 7700 Prism Sequence Detection system (Applied Biosystems, Carlsbad, CA USA). Analyses of the qRT-PCR were performed with SDS 1.2.3 software (Applied Biosystems, Carlsbad, CA USA). All data were normalized to the expression of the housekeeping gene (18SrRNA). The formula for calculating the relative messenger (m) RNA expression from the data was as follows:

Relative mRNA expression: $2^{-(\Delta \Lambda \mathrm{CT})}$

${ }^{\Delta \mathrm{CT}}=\mathrm{CT}$ housekeeping gene $-\mathrm{CT}$ gene of interest

$\mathrm{CT}$ is the threshold cycle (relative measure of the concentration of the target in the $\mathrm{PCR}$ reaction).

\section{Statistical Analysis}

The results were analyzed with $t$-tests (GraphPad Software, Inc., San Diego, CA, USA). All values are stated as mean \pm standard deviation. A $\mathrm{P}$ value of less than 0.05 was considered to indicate statistical significance.

\section{Results}

\section{Levels of Growth Factors in Sera}

Levels of the growth factors PDGF-AB, PDGF-BB, and TGF- $\beta 1$ in all HS $(n=8)$ and FBS $(n=3)$ samples were analyzed with ELISA. The concentration of PDGF-AB in HS (14.095 $\pm 5.310 \mathrm{ng} / \mathrm{mL})$ was significantly greater $(\mathrm{p}<0.05)$ than that in FBS $(0.239$ $\pm 0.092 \mathrm{ng} / \mathrm{mL}$ ). Similarly, the concentration of PDGF-BB was significantly greater $(\mathrm{p}<0.05)$ in HS $(1.185 \pm 0.2783 \mathrm{ng} / \mathrm{mL})$ than in FBS $(0.1840 \pm 0.4808$ $\mathrm{ng} / \mathrm{mL}$ ). Finally, the concentration of TGF- $\beta 1$ in HS $(26.83 \pm 2.083 \mathrm{ng} / \mathrm{mL})$ was significantly greater $(\mathrm{p}<$ 0.05) than that in FBS $(11.18 \pm 0.2791 \mathrm{ng} / \mathrm{mL})$ (Fig. 2 a).

\section{Proliferation of ASCs Was Accelerated in HS-} CM

The ASCs grown in HS-CM showed more rapid 
Table 1 List of primers used in qRT-PCR to identify expression of adipogenic, osteogenic, and chondrogenic differentiation factors

\begin{tabular}{cccc}
\hline Gene & & Primer 5'Sequence-3’ & Accession Number \\
\hline PPAR $\gamma$ & $\mathrm{F}$ & AGGAGCGGGTGAAGACTCAT & NM_015869 \\
& $\mathrm{R}$ & CGAAGCTGCTCCAGAAAATG & \\
RUNX2 & $\mathrm{F}$ & CCCGTGGCCTTCAAGGT & NM_0004348.3 \\
& $\mathrm{R}$ & CGTTACCCGCCATGACAGTA & \\
SPOCK1 & $\mathrm{F}$ & ACATCGGGCCTTGCAAATACA & NM_004598.3 \\
& $\mathrm{R}$ & GAAGCAGCCGGCCCACTCATC & \\
SOX9 & $\mathrm{F}$ & GCGGAGGAAGTCGGTGAAGA & NM_000346.3 \\
& $\mathrm{R}$ & CCCTCTCGCTTCAGGTCAGC & \\
\multirow{2}{*}{ 18SrRNA } & $\mathrm{F}$ & GTAACCCGTTGAACCCCATT & NR_003286.2 \\
& $\mathrm{R}$ & TCTTGGCAGCAGGATAGTCCTT & \\
\hline
\end{tabular}

[a]
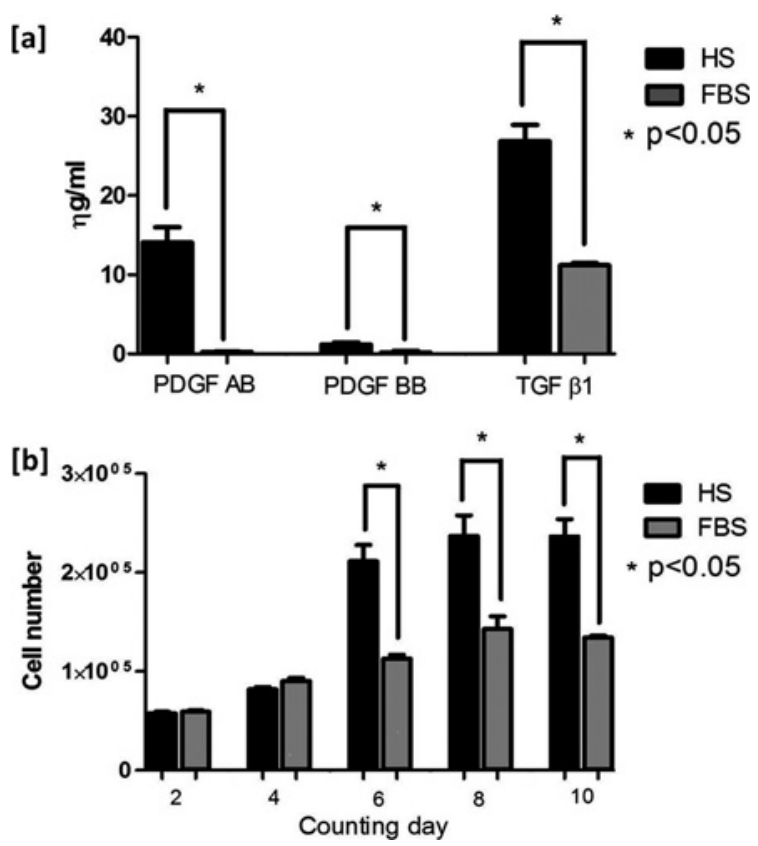

Fig. 2 Growth factor quantification in HS and FBS with ELISA and the proliferation potential of hASCs cultured in HS-CM and FBS-CM. [a] Concentrations of growth factors in HS $(n=8)$ and FBS $(n=3)$. The levels of PDGF$\mathrm{AB}, \quad \mathrm{PDGF}-\mathrm{BB}$, and TGF- $\beta 1$ were significantly higher in HS than in FBS. [b] Cell numbers in each culture condition were counted on days $0,2,4,6,8$, and 10 . The cell numbers were significantly higher in HS$\mathrm{CM}$ culture than in FBS-CM culture on days 6,8 , and $10(\mathrm{p}<0.05)$.

proliferation than did ASCs grown in FBS-CM (Fig. 2b). The differences between HS and FBS were not significant on day 2 of culture $(57,304 \pm 1,828$ cells vs. $59,018 \pm 1,657$ cells, respectively; $\mathrm{p}>0.05$ ) or day 4 of culture $(81,872 \pm 2,198$ cells vs. $90,147 \pm 3,173$ cells; $\mathrm{p}>0.050$ ). However, the numbers of cells in HS-CM were significantly greater than in FBS-CM on day 6 $(211,277 \pm 16,556$ cells vs. $112,885 \pm 4,108$ cells, respectively; $\mathrm{p}<0.05)$, day $8(236,819 \pm 21,096$ cells vs. $142,819 \pm 12,924$ cells; $\mathrm{p}<0.05)$, and day $10(236,293 \pm$ 17,800 cells vs. $134,426 \pm 1,647$ cells; $\mathrm{p}<0.05$ ).

Culture in HS-CM Did Not Alter Cell Surface Markers of ASCs as Detected with FACS Analysis

The hASCs cultured in either HS-CM or FBS-CM showed identical patterns of surface protein expression with no significant variation in surface marker expression among different samples. All samples were positive for the surface proteins CD73, CD90, and CD105 and were negative for CD31, CD34, and CD45 (Fig. 3).

\section{Differentiation Capacities of ASCs}

At the second passage, hASCs cultured in medium supplemented with either HS or FBS were examined for their potential to differentiate into adipogenic, osteogenic, and chondrogenic lineages. A significant fraction of the cells cultured in adipogenic differentiation conditions contained multiple, intracellular lipid-filled droplets, as indicated by Oil red O staining. In all the osteogenically induced cells, calcium deposits were detected with von Kossa staining. All the cells induced to undergo chondrogenic differentiation exhibited pericellular proteoglycan deposition, as indicated by Alcian blue staining (Fig. 4a). 

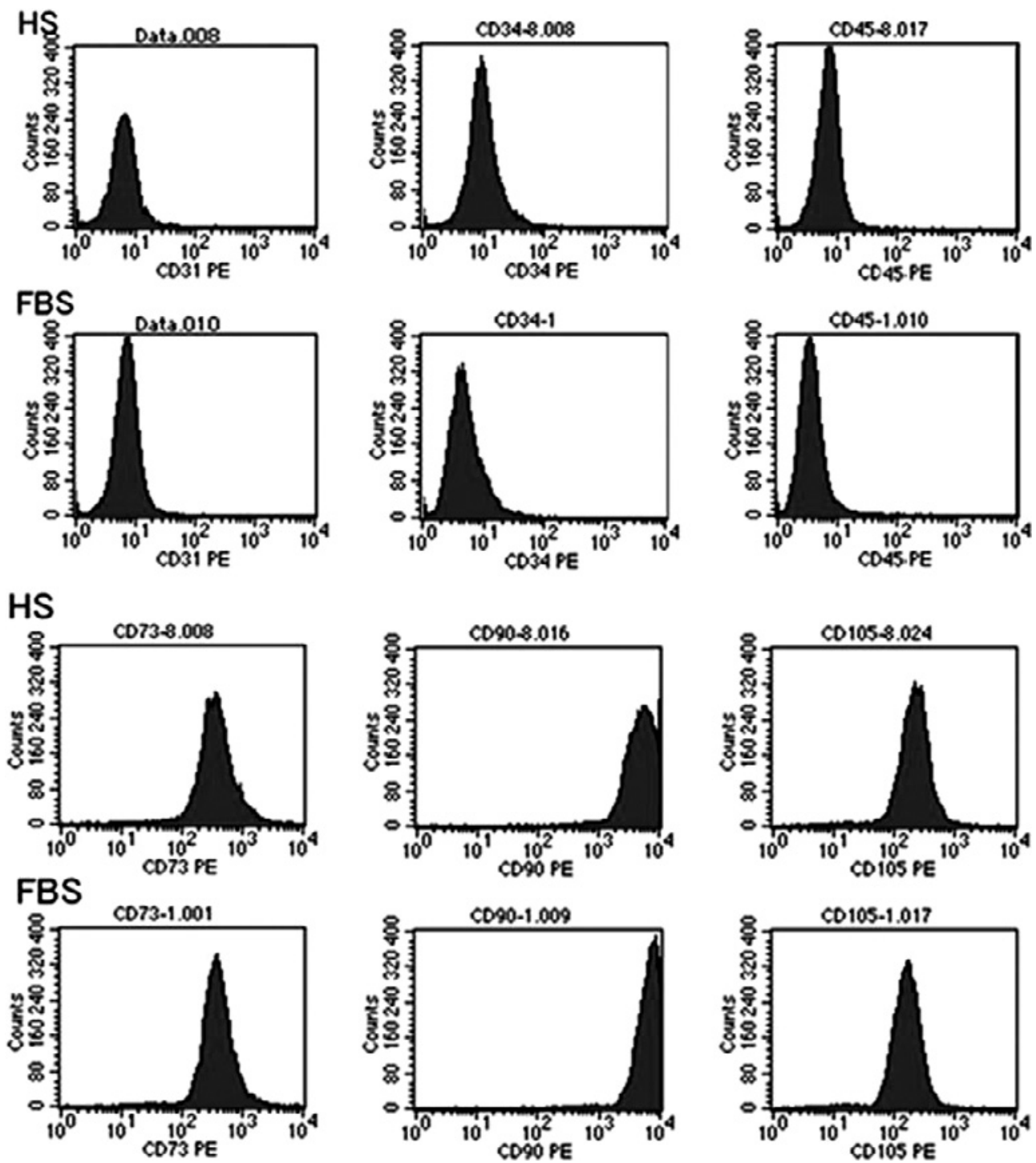

Fig. 3 Representative histograms of flow cytometric analysis of cell surface marker expression. Expression of CD31, CD34, CD45, CD73, CD90, and CD105 was measured in hASCs cultured in HS-CM or FBS-CM at passage 6. Both cells cultured in HS-CM and cells cultured in FBS-CM cultured showed similar patterns of expression: CD31 (-), CD34 (-), CD45 (-), CD73 (+), CD90 (+), and CD105 (+).

HS-supplemented Medium Induced Higher Levels of Gene Expression during Differentiation than FBS as Detected by qRT-PCR

After 2 weeks of adipogenic induction, the expression of PPAR $\gamma$ was upregulated both in cells cultured in HS and in cells cultured in FBS, but no significant difference between HS (1.715 \pm 0.2550) and FBS (1.235 \pm 0.0350, p>0.05) was detected. Expression of 2 osteogenesis-specific genes (RUNX2 and SPOCK1) was analyzed with qRT-PCR after 28 days of osteogenic induction. The expression of RUNX2 and SPOCK1 was upregulated in cells from both HS-supplemented and FBS-supplemented media, and RUNX2 showed significantly higher expression in HS-supplemented medium $(2.08 \pm 0.03)$ than in FBS-supplemented medium $(0.98 \pm 0.185, \mathrm{p}<$ 0.05). Chondrogenesis of ASCs cultured in either HS or FBS was analyzed by quantifying the expression of SOX9. The SOX9 expression after 3 weeks in induction medium was significantly higher in HSsupplemented medium $(11.18 \pm 0.5650)$ than in FBSsupplemented medium $(1.275 \pm 0.2750, \mathrm{p}<0.05)$ (Fig. 4b). 
[a]
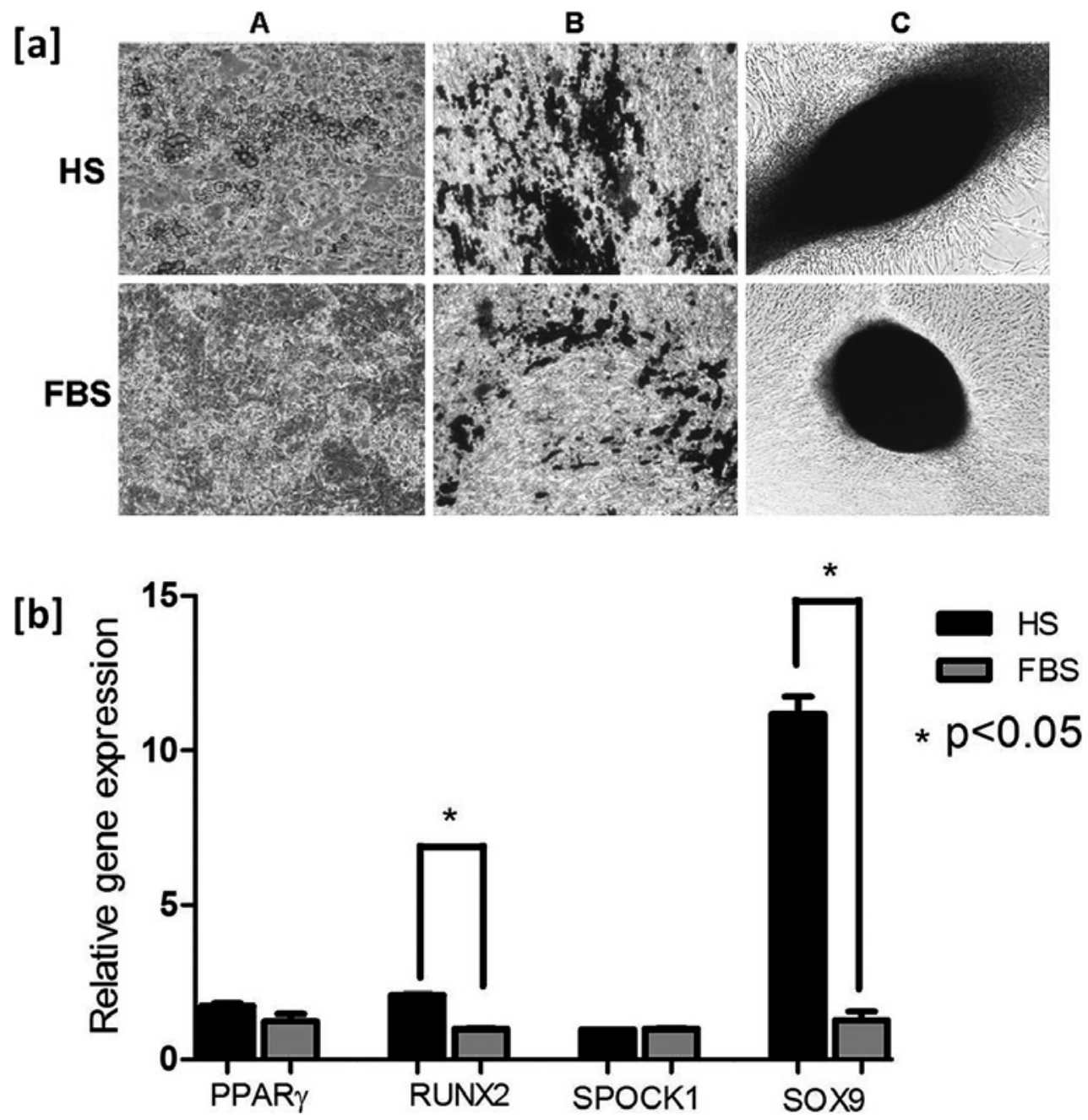

Fig. 4 Comparison of differentiation potential between hASCs cultured in differentiation medium supplemented with HS or FBS. [a] Microscopic results of cell differentiation: (A) adipogenesis (Oil red O staining), (B) osteogenesis (von Kossa staining), and (C) chondrogenesis (Alcian blue staining). Upper panel: cells cultured in HS-supplemented medium. Lower panel: cells cultured in FBS-supplemented medium. All cell populations in both media had similar patterns of differentiation. [b] Adipogenic, osteogenic, and chondrogenic gene expression in ASCs cultured in HS-supplemented induction medium compared with that in FBS-supplemented induction medium. Quantitative RT-PCR showed significantly higher expression of RUNX2 and SOX9 in HS-supplemented osteogenic and chondrogenic induction media $(\mathrm{p}<0.05)$.

\section{Discussion}

A variety of on-going preclinical trials are using hASCs to search for treatments for such conditions as myocardial infarction ${ }^{30-32}$ and liver failure $^{33}$. However, most isolation and expansion protocols for clinical-scale production of ASCs use culture medium supplemented with FBS, which contains xenogeneic proteins that can be internalized by $\mathrm{ASCs}^{34}$. Consequently, a host of potential problems, such as viral and prion transmission and immunological reactions, can arise $\mathrm{e}^{14,35,36}$.

The present results show that cell proliferation was more favorable with HS-CM than with FBS-CM. Sera from 8 different donors all showed favorable results, suggesting that autologous serum would produce similar effects. The accelerated expansion protocol would be highly valuable when the original amount of hASCs is limited, such as cell-based therapy with banked hASCs.

The surface marker expression of hASCs grown with HS-CM was indistinguishable from those cultured with FBS-CM. The expression patterns 
after the sixth passage are similar to those reported previously $^{37}$. These results suggest that HS-CM did not differ from FBS-CM in its effects on differentiation, as evaluated with cell surface markers. Additionally, cells grown in HS were able to differentiate into 3 different lineages-adipogenic, osteogenic, and chondrogenic-like cells grown with FBS. Interestingly, our results revealed that osteogenesis and chondrogenesis showed a significantly higher expression of RUNX2 and SOX9, respectively, suggesting that hASCs cultured in HS have greater differentiation potential for osteogenesis and chondrogenesis than do hASCs cultured in FBS. These results indicate that HS has no adverse effects on the characteristic features or multipotency of hASCs. Taken together, these results suggest HS is more suitable for growing hASCs for clinical applications than is the commonly used conventional methods using FBS.

PDGFs are composed of 2 polypeptide chains (A and $\mathrm{B}$ ) combined in 3 disulfide-linked dimeric forms $(\mathrm{AA}, \mathrm{AB} \text {, and } \mathrm{BB})^{38}$. Human platelets have all 3 PDGF dimers, in quantities of approximately 65\% $\mathrm{AB}, 23 \% \mathrm{BB}$, and $12 \% \mathrm{AA}$. In this study, the concentrations of PDGF-AB and PDGF-BB in HS were evaluated. A previous report has indicated an optimal concentration of PDGF for proliferation of hASCs ${ }^{21}$. The ranges of PDGF-AB and PDGF-BB in HS obtained with Cellaid ${ }^{\circledR}$ in the present study were 7.5 to $24 \mathrm{ng} / \mathrm{mL}$ and 0.2 to $2.4 \mathrm{ng} / \mathrm{mL}$, respectively. Therefore, the concentrations of growth factors in HS-supplemented medium were within the optimal concentration ranges. The proliferation of hASCs and the concentration of these growth factors showed a positive correlation and suggest that the levels of these growth factors have significant effects on ASC proliferation.

The Cellaid ${ }^{\circledR}$ serum collection bag system used in the present study contained glass beads that function as platelet activators. A gentle agitating process over 30 minutes allows the glass beads to remove fibrin from whole blood and to activate platelets. The levels of PDGF-AB, PDGF-BB, and TGF- $\beta 1$ were increased in all sera obtained, compared with those with standard HS collection methods. Because the Cellaid ${ }^{\circledR}$ bag is designed to obtain serum in a completely closed system, the risk of microbial contamination is minimal. The usage of Cellaid $^{\circledR}$ would make it possible to prepare autologous serum containing large amounts of cellular growth factors for cell-based therapy in the future.

\section{Conclusions}

This study has demonstrated that hASCs can be grown rapidly while preserving their multipotency with an HS-supplemented medium. The growth factors derived from platelets in HS enhanced cell proliferation. These results indicate that $\mathrm{HS}$ is a suitable substitute for FBS and can greatly improve the progress of basic research and cell-based therapy.

Conflict of interest: The authors have no financial conflicts of interest regarding the publication of this article.

Acknowledgment: The authors thank Ms. M. Takatori and Mr. H. Takafuji for their excellent technical assistance.

\section{References}

1. Takahashi K, Tanabe K, Ohnuki M, et al.: Induction of pluripotent stem cells from adult human fibroblasts by defined factors. Cell 2007; 131: 861-872.

2. Rafii S, Lyden D: Therapeutic stem and progenitor cell transplantation for organ vascularization and regeneration. Nat Med 2003; 9: 702-712.

3. Wobus AM, Boheler KR: Embryonic stem cells: prospects for developmental biology and cell therapy. Physiol Rev 2005; 85: 635-678.

4. Young FE: A time for restraint. Science 2000; 287: 1424.

5. Lenoir N: Europe confronts the embryonic stem cell research challenge. Science 2000; 287: 1425-1427.

6. Caplan AI: Mesenchymal stem cells. J Orthop Res 1991; 9: 641-650.

7. Bieback K, Kern S, Kluter H, Eichler H: Critical parameters for the isolation of mesenchymal stem cells from umbilical cord blood. Stem Cells 2004; 22 : 625-634.

8. Kern S, Eichler H, Stoeve J, Kluter H, Bieback K: Comparative analysis of mesenchymal stem cells from bone marrow, umbilical cord blood, or adipose tissue. Stem Cells 2006; 24: 1294-1301.

9. Zuk PA, Zhu M, Ashjian P, et al.: Human adipose tissue is a source of multipotent stem cells. Mol Biol Cell 2002; 13: 4279-4295.

10. Zuk PA, Zhu M, Mizuno H, et al.: Multilineage cells 
from human adipose tissue: implications for cellbased therapies. Tissue Eng 2001; 7: 211-228.

11. Tholpady SS, Llull R, Ogle RC, Rubin JP, Futrell JW, Katz AJ: Adipose tissue: stem cells and beyond. Clin Plast Surg 2006; 33: 55-62.

12. Bunnell BA, Flaat M, Gagliardi C, Patel B, Ripoll C: Adipose-derived stem cells: isolation, expansion and differentiation. Methods 2008; 45: 115-120.

13. Katz AJ, Llull R, Hedrick MH, Futrell JW: Emerging approaches to the tissue engineering of fat. Clin Plast Surg 1999; 26: 587-603.

14. Selvaggi TA, Walker RE, Fleisher TA: Development of antibodies to fetal calf serum with arthus-like reactions in human immunodeficiency virus-infected patients given syngeneic lymphocyte infusions. Blood 1997; 89: 776-779.

15. Gregory CA, Reyes E, Whitney MJ, Spees JL: Enhanced engraftment of mesenchymal stem cells in a cutaneous wound model by culture in allogenic species-specific serum and administration in fibrin constructs. Stem Cells 2006; 24: 2232-2243.

16. Chachques JC, Herreros J, Trainini J, et al: Autologous human serum for cell culture avoids the implantation of cardioverter-defibrillators in cellular cardiomyoplasty. Int J Cardiol 2004; 95 Suppl 1: S29S33.

17. Kocaoemer A, Kern S, Kluter H, Bieback K: Human $\mathrm{AB}$ serum and thrombin-activated platelet-rich plasma are suitable alternatives to fetal calf serum for the expansion of mesenchymal stem cells from adipose tissue. Stem Cells 2007; 25: 1270-1278.

18. Kurita M, Aiba-Kojima E, Shigeura T, et al.: Differential effects of three preparations of human serum on expansion of various types of human cells. Plast Reconstr Surg 2008; 122: 438-448.

19. Lindroos B, Aho KL, Kuokkanen H, et al.: Differential gene expression in adipose stem cells cultured in allogeneic human serum versus fetal bovine serum. Tissue Eng Part A 2010; 16: 2281-2294.

20. Bieback K, Ha VA, Hecker A, et al: Altered gene expression in human adipose stem cells cultured with fetal bovine serum compared to human supplements. Tissue Eng Part A 2010; 16: 3467-3484.

21. Kakudo N, Minakata T, Mitsui T, Kushida S, Notodihardjo FZ, Kusumoto K: Proliferationpromoting effect of platelet-rich plasma on human adipose-derived stem cells and human dermal fibroblasts. Plast Reconstr Surg 2008; 122: 1352-1360.

22. Doucet C, Ernou I, Zhang Y, et al.: Platelet lysates promote mesenchymal stem cells expansion: A safety substitute for animal serum in cell-based therapy applications. J Cell Physiol 2005; 205: 228236.

23. Mizuno N, Shiba H, Ozeki Y, et al:: Human autologous serum obtained using a completely closed bag system as a substitute for foetal calf serum in human mesenchymal stem cell cultures. Cell Biol Int 2006; 30: 521-524.

24. Nimura A, Muneta $T$, Koga $H$, et al.: Increased proliferation of human synovial mesenchymal stem cells with autologous human serum: comparisons with bone marrow mesenchymal stem cells and with fetal bovine serum. Arthritis Rheum 2008; 58: 501510 .
25. Isogai N, Nakagawa Y, Suzuki K, et al.: Cytokine-rich autologous serum system for cartilaginous tissue engineering. Ann Plast Surg 2008; 60: 703-709.

26. Tobita M, Uysal AC, Ogawa R, Hyakusoku H, Mizuno H: Periodontal tissue regeneration with adipose-derived stem cells. Tissue Eng Part A 2008; 14: 945-953.

27. Ogawa R, Mizuno H, Watanabe A, Migita M, Shimada T, Hyakusoku H: Osteogenic and chondrogenic differentiation by adipose-derived stem cells harvested from GFP transgenic mice. Biochem Biophys Res Commun 2004; 313: 871-877.

28. Lu F, Mizuno H, Uysal AC, Cai X, Ogawa R, Hyakusoku H: Improved viability of random pattern skin flaps through the use of adipose-derived stem cells. Plast Reconstr Surg 2008; 121: 50-58.

29. Denker AE, Nicoll SB, Tuan RS: Formation of cartilage-like spheroids by micromass cultures of murine C3H10T1/2 cells upon treatment with transforming growth factor-beta 1. Differentiation 1995; 59: 25-34.

30. Miyahara $\mathrm{Y}$, Nagaya $\mathrm{N}$, Kataoka $\mathrm{M}$, et al: Monolayered mesenchymal stem cells repair scarred myocardium after myocardial infarction. Nat Med 2006; 12: 459-465.

31. Fraser JK, Schreiber R, Strem B, et al.: Plasticity of human adipose stem cells toward endothelial cells and cardiomyocytes. Nat Clin Pract Cardiovasc Med 2006; 3: S33-S37.

32. Planat-Benard V, Menard C, Andre M, et al.: Spontaneous cardiomyocyte differentiation from adipose tissue stroma cells. Circ Res 2004; 94: 223229 .

33. Banas A, Teratani T, Yamamoto Y, et al.: Rapid hepatic fate specification of adipose-derived stem cells and their therapeutic potential for liver failure. J Gastroenterol Hepatol 2009; 24: 70-77.

34. Spees JL, Gregory CA, Singh H, et al: Internalized antigens must be removed to prepare hypoimmunogenic mesenchymal stem cells for cell and gene therapy. Mol Ther 2004; 9: 747-756.

35. Tuschong L, Soenen SL, Blaese RM, Candotti F, Muul LM: Immune response to fetal calf serum by two adenosine deaminase-deficient patients after $\mathrm{T}$ cell gene therapy. Hum Gene Ther 2002; 13: 16051610.

36. Drach G, Maret A, Richard MF, Barbu E: Transfer and induction of delayed hypersensitivity to methylated bovine serum albumin in the absence of adjuvant. C R Acad Sci Hebd Seances Acad Sci D 1977; 284: 2435-2437.

37. Suga H, Shigeura T, Matsumoto D, et al.: Rapid expansion of human adipose-derived stromal cells preserving multipotency. Cytotherapy 2007; 9: 738745.

38. Hart CE, Bailey M, Curtis DA, et al.: Purification of PDGF-AB and PDGF-BB from human platelet extracts and identification of all three PDGF dimers in human platelets. Biochemistry 1990; 29: 166-172.

(Received, September 11, 2012) (Accepted, October 18, 2012) 